\title{
UNA PROPUESTA PARA LA DIDÁCTICA DE LA EDUCACIÓN AMBIENTAL EN LAS
}

UNIVERSIDADES PÚBLICAS

Lisbeth Bermudez y Dilia Escalante de Urrecheaga

(pp 102-124)

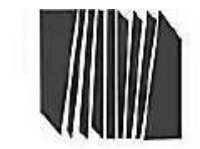

UNIVCREIDAD PCOACOGICA EXP CRIMCNTALL LIOCRTAOO O

\section{Revista}

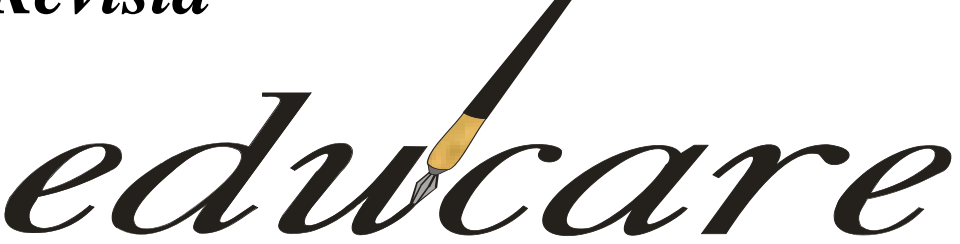

Órgano Divulgativo de la Subdirección de Investigación y Postgrado del Instituto Pedagógico de Barquisimeto "Luis Beltrán Prieto Figueroa"

\section{Volumen 22 № 1}

Volumen 22 № 1
UNA PROPUESTA PARA LA DIDÁCTICA DE LA EDUCACIÓN AMBIENTAL EN LAS
UNIVERSIDADES PÚBLICAS
A PROPOSAL FOR THE TEACHING OF ENVIRONMENTAL EDUCATION IN PUBLIC
UNIVERSITIES
I S S N 2018 . 4 - 7296

\author{
AUTOR \\ LISBETH BERMÚDEZ \\ UNIVERSIDAD NACIONAL EXPERIMENTAL DE LA FUERZA \\ ARMADA (UNEFA) \\ DILIA ESCALANTE DE URRECHEAGA \\ UNIVERSIDAD DE LOS ANDES (ULA)
}

Volumen $22 N^{\circ} 1$ Enero-Abril 2018. ISSN: 2244-7296 


\section{Propuesta Educativa}

Recibido
Lisbeth Bermúdez Lunar*

Dilia Escalante de Urrecheaga**

Aceptado

\section{RESUMEN}

La utilización incontrolada de la ciencia y la tecnología ha impactado el ambiente, originando desequilibrios en el entorno vinculados con la interacción hombrenaturaleza. El presente artículo en la modalidad de propuesta educativa tiene como propósito ofrecer a los docentes universitarios acciones estratégicas-pedagógicas para la asignatura de Educación Ambiental a fin de desarrollar en los estudiantes valores fundamentales que mejoren la interacción del hombre con el ambiente. El trabajo se enmarca en un análisis crítico-reflexivo de una investigación cualitativa - cuantitativa previa (Bermúdez, Escalante y Figueira, 2016), cuyos resultados evidenciaron la necesidad de reorientar la praxis de la Educación Ambiental, lo cual permitió formular una plan de acciones para desarrollar esta asignatura de una manera transformadora. La propuesta se fundamenta en la responsabilidad social que la universidad pública venezolana, específicamente del Estado Nueva Esparta debe asumir al promover la participación activa de los estudiantes en la solución de los problemas ambientales de su comunidad

Descriptores: educación ambiental, propuesta didáctica, educación universitaria

\begin{abstract}
The uncontrolled use of science and technology has negatively impacted the environment; it has generated imbalances in the environment related to the interaction between man and nature. The present article is an educational proposal, which has the purpose of offering strategic and pedagogical actions to professors at the university levels for the Environmental Education course. It also aims to develop students' fundamental values in order to increase the interaction of the man with the environment. This paper is a critical-reflexive analysis to a previous qualitative-quantitative research (Bermúdez, Escalante y Figueira, 2016), whose results showed the necessity of reorienting the Environmental Education praxis and also allowed to design an action plan to the corse mentioned above in a transforming way. This proposal is based on the social responsibility that Venezuelan Public University should take, specifically at Nueva Esparta State, in the promotion of an active participation of the students to solve their community environmental problems.
\end{abstract}

Key words: Environmental Education, educational proposal, public university educational level

\footnotetext{
* Lisbeth Natividad Bermúdez Lunar, Licenciada en Educación mención Biología y Química, Maestría en Educación Ambiental. Docente Instructor de la Universidad Nacional Experimental de la Fuerza Armada (UNEFA), lisbethbermudez.postgradounefa@gmail.com.

${ }^{* *}$ Licenciada en Educación mención Inglés. Maestría en Lectura. Profesora Titular Jubilada de la Universidad de Los
Andes con Doctorado (PhD) en el área de Alfabetización y Lenguaje. Correo electrónico diliaesc@ @ hotmail.com
}

Volumen $22 N^{\circ} 1$ Enero-Abril 2018. ISSN: 2244-7296 


\section{UNA PROPUESTA PARA LA DIDÁCTICA DE LA EDUCACIÓN AMBIENTAL EN LAS}

UNIVERSIDADES PÚBLICAS

Lisbeth Bermudez y Dilia Escalante de Urrecheaga

(pp 102-124)

\section{INTRODUCCIÓN}

La revolución industrial se caracterizó por profundos avances tecnológicos, cambios ideológicos, políticos y socioeconómicos que desarrolló un modelo económico basado en el aumento de la producción a menor costo por parte de los países industrializados, consolidando de esta manera el capitalismo, el cual muchos autores consideran la génesis de los problemas ambientales.

Esta situación trajo consigo un estilo de vida que se fundamentaba en la explotación de los recursos naturales de los países subdesarrollados, convirtiéndose en un saqueo a la naturaleza y afectando la coexistencia armónica entre el hombre y su ambiente. Consecuencia de ello, es reflejado en los problemas ambientales que actualmente existen en todo el planeta, donde el hombre ha hecho uso desmedido de la naturaleza rompiendo la dinámica y equilibrio que debe existir para evitar consecuencias que afectan directamente la preservación de la vida en el planeta.

Tal hecho ha enaltecido los valores de una sociedad caracterizada por el consumismo, aumento de la riqueza y el confort; y el fortalecimiento de un modelo de desarrollo que tiene como visión el dominio del hombre sobre la naturaleza, ya que ésta es manipulada como una mercancía, sin tomar en cuenta el valor ético; tal como lo afirma Velásquez y Fernández (1998) cuando expresan: "la importancia que desde entonces tendrá la acumulación de capital para sus poseedores irá convirtiendo en objetos al hombre y la naturaleza que pasarán a ser considerados como mercancías" (p.12). Este cambio en la concepción del bienestar del hombre fue agravando el deterioro ambiental debido a que comenzó a fomentarse una sociedad de consumo.

Como evidencias de esta manipulación se tiene la desertificación de tierras aptas para el cultivo, los patrones de asentamiento y ocupación del espacio, la contaminación de aguas, aire y suelo por el uso y abuso indiscriminado de gases y pesticidas, la deforestación de grandes áreas boscosas, el calentamiento global y el cambio climático que en gran medida 


\section{UNA PROPUESTA PARA LA DIDÁCTICA DE LA EDUCACIÓN AMBIENTAL EN LAS}

UNIVERSIDADES PÚBLICAS

Lisbeth Bermudez y Dilia Escalante de Urrecheaga

(pp 102-124)

afecta a todo el planeta hoy en día. Todas estas actividades humanas comenzaron a producir alteraciones visibles en el ambiente debido a los cambios de estilo de vida del hombre; tal como lo refleja la afirmación de Leff (2004) cuando señala que:

La interpretación recíproca de naturaleza y sociedad no es considerada aquí como la articulación o interdeterminación entre procesos naturales y procesos sociales, sino que se reduce a la interiorización del mundo por la praxis humana que constituye un 'todo natural' (p.23).

Ante esta realidad surge la necesidad de abordar la interacción sociedad-naturaleza, considerando el papel dinamizador del ser humano en su doble condición de elemento natural y social, ya que de su actuación depende que el efecto de esas interrelaciones sea una forma sostenible de desarrollo, que se traduzca en una mejor calidad de vida; de ahí, la inquietud de enfrentar dicha problemática desde todos los ámbitos y entes de la sociedad.

Así, la educación, surge como la vía más acorde para lograr el cambio de conducta y de concepción hacia la naturaleza, para buscar soluciones a los problemas ambientales que alarman a la humanidad, como son el efecto invernadero, cambio climático, aumento de la pobreza, marginalidad, entre otros. En lo particular, la universidad pública tiene la responsabilidad de interceder y asumir los problemas ambientales concatenados con los procesos de transformación de la sociedad, no desde una visión unidisciplinaria que enmascara la correlación de los problemas y situaciones, sino desde una perspectiva interdisciplinaria, y más aún transdisciplinaria.

En este sentido, se hace necesario una revolución del pensamiento, un cambio de la visión del mundo y la naturaleza, y una transformación del conocimiento. Para lograrlo se requiere de una nueva praxis educativa, que construya un nuevo saber y una nueva racionalidad, donde la educación deje de seguir reforzando y reproduciendo un modo de vida cuya lógica de la rentabilidad económica subordine el interés colectivo por el mejoramiento de la calidad de vida humana y la salud ambiental a los intereses individuales o de grupos económicos. De ahí, que la universidad debe ser un espacio de revolución de 


\section{UNA PROPUESTA PARA LA DIDÁCTICA DE LA EDUCACIÓN AMBIENTAL EN LAS}

UNIVERSIDADES PÚBLICAS

Lisbeth Bermudez y Dilia Escalante de Urrecheaga

(pp 102-124)

los saberes y conocimientos donde se deje de considerar la idea de la naturaleza como simple medio de obtención de bienes y servicio; sobre todo en Venezuela, que por su ubicación geográfica en el Mar Caribe posee grandes atractivos turísticos que impresionan la imaginación y las cuales, en el transitar del viajero, pueden llevar desde elevados páramos de gélidas cumbres, pasar por intrincadas selvas esmeralda de naturaleza inigualables y llegar a exuberantes playas cristalinas. Y, en este tránsito encontrar lugares históricos de renombre y gente cálida que consolidan la proyección internacional del país como es el caso del estado Nueva Esparta.

Por lo antes expuesto, a fin de mantener la belleza natural con que ha sido bendecida Venezuela y remediar los desaciertos que de manera voluntaria o involuntaria se cometen, se presenta una Propuesta para la Didáctica de la Educación Ambiental dirigida a las instituciones públicas de educación universitaria del estado Nueva Esparta. Se estima que la misma permite redimensionar los contenidos de educación ambiental que ofrecen sus planes de estudio hacia actividades interdisciplinarias y transdisciplinarias que vinculan experiencias del campo técnico, científico y humanístico.

\section{LA EDUCACIÓN AMBIENTAL COMO AGENTE TRANSFORMADOR}

La segunda mitad del siglo XVIII se caracterizó por grandes innovaciones e ideas que continuaron manifestándose en el tiempo de manera paralela al crecimiento de una economía basada en el capitalismo industrial. Arana (1999) recuerda la trascendencia de tal hecho cuando señala que:

Bajo el contexto de la crisis moderna de la civilización industrial se desarrolla la llamada tercera revolución científica-tecnológica, encabezada por la microelectrónica, la tecnología de nuevos materiales y las biotecnologías, de las cuales se espera un profundo impacto sobre los procesos de desarrollo y consecuentemente, cambio en las estructuras socioeconómicas a nivel mundial. (p.53)

Volumen $22 N^{\circ} 1$ Enero-Abril 2018. ISSN: 2244-7296 
UNA PROPUESTA PARA LA DIDÁCTICA DE LA EDUCACIÓN AMBIENTAL EN LAS

UNIVERSIDADES PÚBLICAS

Lisbeth Bermudez y Dilia Escalante de Urrecheaga

(pp 102-124)

Como consecuencia de esta realidad, parafraseando lo que expresa Arana (ob.cit) se considera que países en vías de desarrollo como Venezuela deben enfrentar dos problemas primarios: 1) luchar por obtener una identidad propia y sentido de pertenencia frente a un proceso de globalización económica del cual no puede ser excluida, y debe enfrentar; y 2) construir un modelo de desarrollo propio e independiente donde no se pierda su idiosincrasia que lo caracteriza como pueblo.

Todo esto indica que las universidades tienen un papel relevante ya que a pesar de la revolución ideológico-política que se refleja en algunos estados del continente americano, en Latinoamérica se sigue evidenciando un capitalismo periférico, crisis presupuestaria y, más grave aún, una educación mediatizada, que tiene como función mantener la cultura consumista en menoscabo de la naturaleza.

Dentro de este contexto, el sistema educativo no ha podido frenar el deterioro ambiental que poco a poco han ido generando las acciones humanas debido a los avances científicos y tecnológicos; ante este panorama, se hace imprescindible la búsqueda de acciones que permitan a los ciudadanos reflexionar y concienciarse en torno a los problemas ambientales que las personas deben enfrentar por la irresponsabilidad en la explotación de la naturaleza. Esta necesidad es expuesta por Caride (2000) al afirmar que la educación ambiental surge

... como propuesta y respuesta educativa para un desarrollo que provea un presente - futuro sustentable; a lo que ha de añadirse, en los términos que sugiere Sauvé (1999), la necesidad de involucrarla en un planteamiento más comprehensivo y congruente con el desarrollo de sociedades responsables, profundizando en las exigencias que ello implica para una reconstrucción armónica de la compleja red de relaciones que existen entre individuos, sociedad y ambiente: de los seres humanos consigo mismo, dentro de sociedades y entre sociedades (p.4)

Así, la educación ambiental es valorada no sólo para sensibilizar, sino también como estrategia para modificar actitudes que propicien conocimientos y criterios para una evolución gradual sobre la concepción que se tiene de la naturaleza, y que ayude al fortalecimiento de una conciencia y ética ecológica en el plano mundial. Es por ello que

Volumen $22 N^{\circ} 1$ Enero-Abril 2018. ISSN: 2244-7296 


\section{UNA PROPUESTA PARA LA DIDÁCTICA DE LA EDUCACIÓN AMBIENTAL EN LAS}

UNIVERSIDADES PÚBLICAS

Lisbeth Bermudez y Dilia Escalante de Urrecheaga

(pp 102-124)

Morín (1993) expresa que la educación refleja una “... inteligencia parcelarizada, compartimentada, mecanicista, reduccionista, rompe el complejo del mundo en fragmentos desglosados, fracciona los problemas, separa lo que está unido unidimensionaliza lo multidimensional" (p.196). De ahí que, la interdisciplinariedad se valora a través de la reflexión amplia y crítica de la realidad; en este caso, de la praxis educativa y la formación de los ciudadanos.

Para ello, es fundamental reorientar las funciones de la educación universitaria que conlleven a la formulación y desarrollo de nuevos paradigmas de pensamiento y acción en la solución de problemas ambientales; teniendo presente que es indispensable que la educación ambiental se desarrolle no como una disciplina más, sino que por el contrario se puedan conjugar varias disciplinas en función de buscar alternativas de solución a los problemas que afectan a la sociedad. Es decir, proponer opciones viables que puedan dar respuesta a la crisis ecológica mundial a través de la praxis de la educación ambiental incorporando la pluralidad axiológica, transformación del conocimiento que en su conjunto puedan revertir la realidad de la interacción hombre - naturaleza.

Ello supone que la educación universitaria debe responder a un todo social, haciendo más amplio su escenario de acción, pues ella constituye un ente legitimador y reproductor de cultura e ideologías que puedan propiciar posibilidades reales de transformar la sociedad y el pensamiento de sus ciudadanos. La universidad tiene que insertarse en la propia dinámica de los inevitables cambios sociales, ambientales, científicos, tecnológicos, de manera que sus orientaciones epistemológicas no se desvanezcan o se superpongan en otras direcciones. Para lo cual, según Hidalgo (2013) se deben abordar

... aspectos que dan coherencia a la actuación y elevan la ética ambiental que se necesita, tales son la pertinencia y contextualización al momento de encaminar acciones. En tal sentido desde la investigación el ambiente debe ser integrado como requisito fundamental en todo proyecto de investigación, desde el propio impacto de su ejecución como del alcance o implicaciones ambientales que pueda tener... Igualmente debe ser asumido en la función de extensión que nos llama a actuar con pertinencia ambiental en la diversidad de espacios sociales

Volumen $22 N^{\circ} 1$ Enero-Abril 2018. ISSN: 2244-7296 
que requieren de los aportes institucionales, en tal sentido debemos participar activamente en su solución. ... La función de docencia que es la razón de ser de las universidades para formar profesionales integrales, debe incorporar la dimensión ambiental en forma transversal en los currículos y no como asignaturas aisladas en el programa de formación profesional. Es claro que pasar de lo tácito a la realidad requiere de la voluntad de sus actores, por lo tanto, los docentes deben ser sensibilizados ambientalmente, y así logren una praxis educativa ambiental de manera natural. (p.4).

Por tal razón, las universidades venezolanas deben impulsar la construcción de una orientación epistemológica donde el orden social, la dinámica natural y el orden simbólico se articulen de tal manera que contribuyan efectivamente a la solución de problemas concretos, dejando de ser un sitio donde se transmiten conocimientos alejados de la realidad; es decir, de la sociedad. De ahí que, según expresa Caride (2000):

No se reduce a educar para "conservar la naturaleza", "concienciar personas" o "cambiar conductas". Su tarea es más profunda y comprometida: educar para cambiar la sociedad, procurando que la toma de conciencia se oriente hacia un desarrollo que sea simultáneamente causa y efecto de la sustentabilidad y la responsabilidad global; [...] promotora de valores que inciten la transformación social, el pensamiento crítico y la acción emancipatoria (p.6).

De lo anterior se deduce la incidencia que tiene la educación ambiental en diferentes enfoques: a) sociales, busca minimizar o solucionar problemas de pobreza y marginalidad; b) ambientalista, tiende al mantenimiento y conservación de la naturaleza; c) urbanístico, sugiere la ordenación del territorio de tal manera que no dañe el ambiente; y d) culturalideológico, está fundamentada en la formación de valores y ética ambiental que contrarreste los valores y cultura del capitalismo dominante. Es decir, el sistema educativo universitario debe responder a todos estos enfoques que en su conjunto logren la transformación al pensamiento crítico de todos los estudiantes.

Este clamor en el sector universitario adquiere rango constitucional al indicarse en el artículo 107 de la Constitución de la República Bolivariana de Venezuela que "la educación ambiental es obligatoria en los niveles y modalidades del sistema educativo, así como también en la educación ciudadana no formal. Es de obligatorio cumplimiento en las 
instituciones públicas y privadas”. Así mismo, se le asigna responsabilidad en la Ley del Plan de la Patria, Segundo Plan Socialista de Desarrollo Económico y Social de la Nación, 2013-2019, en su quinto objetivo histórico: “Contribuir con la preservación de la vida en el planeta y la salvación de la especie humana, y el objetivo estratégico general 5.1.2.4, donde se resalta: "Fomentar un nuevo esquema de valores, orientado al respeto y preservación de la naturaleza, que transforme la conciencia colectiva, sobre los patrones capitalistas de producción y consumo".

La incorporación de la educación ambiental en los planes de estudio universitarios además se fundamenta en acuerdos internacionales como la Declaración de Aichi-Nagoya sobre la Educación para el Desarrollo Sostenible (UNESCO, 2014); y que se reflejan en las ideas de Révora (2001) al señalar que la formación ambiental universitaria es

... la capacitación de recursos humanos calificados, para encarar la resolución de problemas concretos que afectan a la comunidad local, provincial y nacional con una conciencia profesional y pública fundada en el respeto de los derechos de los distintos actores sociales, sus formas asociativas e institucionales y el compromiso con el desarrollo sustentable. (p. 5).

En este sentido, la intervención de la participación ciudadana en la búsqueda de soluciones a problemas sociales, particularmente en el área ambiental, es ineludible a fin de superar o minimizar el deterioro ambiental acelerado que afecta a Venezuela, casi de manera generalizada. De ahí, vale preguntarnos ¿qué mejor lugar para la participación que la universidad pública, formadora de profesionales quienes se convertirían en multiplicadores de acciones ambientalistas en sus sitios de trabajo y comunidad? Pero para ello, se hace necesario, en palabras de Melendro, Novo, Murga y Bautista (2009) “... dar forma a una Universidad más consciente de su relación con el entorno, difusora de conocimientos vigorosos y contrastados acerca de nuestra realidad ambiental, e implicada en el desarrollo de alternativas para una sociedad sostenible" (s.p.)

Ante esta realidad, se realizó un análisis crítico - reflexivo del alcance conceptual y operación de los aspectos epistemológicos, axiológicos y pedagógicos que evidencian los 
UNA PROPUESTA PARA LA DIDÁCTICA DE LA EDUCACIÓN AMBIENTAL EN LAS

UNIVERSIDADES PÚBLICAS

Lisbeth Bermudez y Dilia Escalante de Urrecheaga

(pp 102-124)

programas de Educación Ambiental que se ofrecen en las carreras de formación docente en las universidades públicas del estado Nueva Esparta, Venezuela (Bermúdez, Escalante y Figueira, 2016). El diseño del estudio fue documental y de campo, bajo un enfoque cualitativo - cuantitativo, con estudiantes que cursaban Educación Ambiental en la Universidad de Oriente (UDO), Universidad Nacional Abierta (UNA), Universidad Nacional Experimental Politécnica de la Fuerza Armada (UNEFA), y Universidad Pedagógica Experimental Libertador (UPEL) y docentes que facilitaban la asignatura.

La muestra estuvo representada por cuatro (4) profesores que facilitaban la asignatura de educación ambiental de las universidades en estudio (UDO, UNA, UPEL, UNEFA) y una muestra probabilística de noventa (90) estudiantes cursantes de la materia. Para la recolección de información se realizaron entrevistas semiestructuradas a los facilitadores de la asignatura de educación ambiental, orientada a darle respuesta a los objetivos planteados en la investigación (Martínez, 2000); además, se aplicó un cuestionario contentivo de 28 preguntas cerradas a los estudiantes cursantes de la asignatura.

En ese estudio se evidenció que los fundamentos epistemológicos, axiológicos y pedagógicos de la educación ambiental en esas instituciones se basan en la mera reformulación de lo conceptual, y que la asignatura es una adición al curriculum “... sin una visión diferente al resto, por lo cual su esencia disciplinaria como medio para cambiar conductas ambientales se ha perdido y su objetivo principal es omitido" (Bermúdez, Escalante y Figueira, 2016; p. 93).

Las conclusiones de la investigación mencionada condujeron al diseño de la Propuesta de Acciones Estratégicas - Pedagógicas para la didáctica de la asignatura de Educación Ambiental en las instituciones públicas de educación universitaria del estado Nueva Esparta, que se presentan en este artículo. Estas estrategias puedan adaptarse a la dinámica social que urge de un cambio en la praxis educativa universitaria en el ámbito ambiental, y que de alguna manera den respuesta a los problemas que aqueja a las comunidades y a los cuales la universidad debe dar respuesta de una manera dinámica y en consonancia con los 


\section{UNA PROPUESTA PARA LA DIDÁCTICA DE LA EDUCACIÓN AMBIENTAL EN LAS}

UNIVERSIDADES PÚBLICAS

Lisbeth Bermudez y Dilia Escalante de Urrecheaga

(рp 102-124)

avances de la sociedad. En tal sentido, la presente propuesta va dirigida a profesionales en formación de cualquier especialidad, carrera o campo de actuación, dado el impacto que como sujetos de un contexto social tienen en su entorno natural, el ambiente; y que van a servir de multiplicadores de conductas con su accionar diario.

\section{LA EDUCACION AMBIENTAL EN EL CONTEXTO UNIVERSITARIO}

\section{Fundamentación pedagógica}

La incorporación de la Educación Ambiental en el currículo es planteada como una necesidad que debe ir más allá de los simples "añadidos" de temas ambientales a los contenidos, pues el reto consiste en integrarla como un elemento dinamizador que obliga al sistema curricular a ajustarse o reorganizarse en función de los objetivos ambientales que se persiguen. Esto traería como consecuencia, un cambio en la praxis educativa que tiene lugar en contextos académicos (i.e. simplificación de la realidad, explicaciones aisladas, parceladas y reducidas); aspectos que no favorece el desarrollo del conocimiento integrado, que permita interpretar las situaciones ambientales en toda su complejidad.

Recordar que cualquier problema ambiental plantea no sólo una visión ecológica sino también una postura ética, económica, política, social, cultural, entre otros; siendo necesario que en su interpretación confluyan disciplinas tan diversas que su análisis pueda enmarcarlas a todas en su justa dimensión; y bajo un marco didáctico que integre reflexión y acción. Las instituciones de educación universitaria deben, mayormente, asumir el compromiso de definir y poner en práctica estrategias educativas que logren concertar acuerdos entre lo ecológico, económico, social, tecnológico y cultural, de tal manera que los ciudadanos y ciudadanas tengan una nueva visión de desarrollo económico global, donde intervienen factores que permitan avanzar hacia un modo de vivir que no comprometa el futuro de las nuevas generaciones. Para ello se hace necesario trabajar la 


\section{UNA PROPUESTA PARA LA DIDÁCTICA DE LA EDUCACIÓN AMBIENTAL EN LAS}

UNIVERSIDADES PÚBLICAS

Lisbeth Bermudez y Dilia Escalante de Urrecheaga

(pp 102-124)

complejidad ambiental a partir de la relación entre áreas que comúnmente están separadas, a fin de replantear o generar conocimientos, y teniendo presente lo expresado por Leff en la Conferencia Magistral: Educación ambiental y filosofía de vida y construcción de mundos sustentables (Universidad de Guanajuato, 2014)

... el problema está en cómo concebir el ambiente para hacer educación ambiental porque una vez que el ambiente manifestó su lado más crítico, empezó toda una reacción en el mundo no solamente de los economistas, no solamente de las ciencias establecida, una reacción en el campo educativo también, es decir una resistencia y una dificultad para enfrentar en toda su criticidad esta crisis ambiental, entonces empezaron toda una serie de estrategias para redefinir lo ambiental para readaptarlo nuevamente a las sinercias del desarrollo establecidas y eso es parte de las grandes dificultades no solamente de la educación ambiental...

La educación en general, y la educación universitaria en particular, tiene sentido en la medida en que forman conocimientos, habilidades y valores orientados a colaborar en la comprensión y solución de los problemas de la sociedad en la que se encuentra inmersa. De allí que, el problema de la degradación ambiental se constituye en un reto para las universidades, pues éste es el sitio más idóneo donde se pueden generar acciones orientadas a la comprensión de dicho desafío.

Ha llegado el momento de dejar de concebir la educación como el poder que tiene el docente de manipular y domesticar a los estudiantes, ya que lo único que esta educación ha conseguido es crear un educando o un profesional castrado y alienado; a quien se le ha negado la posibilidad de pensar y ser partícipe directo de la transformación de su entorno social. Por tanto, el docente debe poseer el conocimiento requerido para poder alcanzar en sus estudiantes el objetivo de desarrollar las habilidades, aptitudes y actitudes que aunado al conocimiento adquirido puedan comprender la verdadera relación equilibrio y dinámica que debe existir entre el hombre y la naturaleza.

De allí que el docente debe tener la capacitación y dominio en el campo ambiental, pero a su vez tener el conocimiento y estrategias que le ayuden a relacionar este saber con otras

Volumen $22 N^{\circ} 1$ Enero-Abril 2018. ISSN: 2244-7296 


\section{UNA PROPUESTA PARA LA DIDÁCTICA DE LA EDUCACIÓN AMBIENTAL EN LAS}

UNIVERSIDADES PÚBLICAS

Lisbeth Bermudez y Dilia Escalante de Urrecheaga

(pp 102-124)

áreas; es decir, lograr que los educandos visualicen los problemas ambientales desde un enfoque transdisciplinario. Por ende, ello trae como consecuencia, la necesidad impostergable de revisar los paradigmas de la comprensión del mundo y del ejercicio docente; así como, el análisis de la problemática ambiental como efecto de un modo de organización social que ha llevado a los seres humanos a un desequilibrio ecológico y social, obligando la transformación de la relación hombre-naturaleza. Hay que definir el quehacer pedagógico si se quiere lograr una formación ambiental, y revisar la práctica educativa desde una perspectiva filosófica y ética que haga ver la necesidad de replantear de fondo el trabajo universitario y su funcionalidad dentro de una sociedad.

Es por ello, que resalta la importancia del señalamiento de Leff (1998), al afirmar que “La educación ambiental implica un proceso más orgánico y reflexivo de reorganización del saber y de la sociedad en la construcción de nuevas capacidades para comprender e intervenir en la transformación del mundo". (p.215). Postura que mantuvo este autor en la Conferencia Magistral: Educación ambiental y filosofía de vida y construcción de mundos sustentables (Universidad de Guanajuato, 2014). Esta situación llama a la reflexión, ya que pasan los años, la situación empeora y las universidades, en sus aulas, mantienen su praxis pedagógica estática. Así que, se hace necesario que el proceso de enseñanza y aprendizaje de la Educación Ambiental en las universidades públicas del estado Nueva Esparta no se oriente sólo hacia la acumulación de conocimientos, sino que esta experiencia de aprendizaje ayude a transformar de manera positiva la visión y la actitud de los profesionales en formación en relación con su entorno. Es decir, sus acciones ambientales deben ser reflejos de un acto de responsabilidad y compromiso con él y la sociedad donde estén inmersos.

\section{Fundamentación curricular}

El currículum debe responder oportuna y permanentemente a la visión, misión, valores y propósitos de las instituciones que lo hacen posible, ofrecer soluciones pertinentes y 


\section{UNA PROPUESTA PARA LA DIDÁCTICA DE LA EDUCACIÓN AMBIENTAL EN LAS}

UNIVERSIDADES PÚBLICAS

Lisbeth Bermudez y Dilia Escalante de Urrecheaga

(pp 102-124)

comprehensivas a los problemas y situaciones de su ámbito local, regional y nacional; así como, mantener un compromiso de calidad y renovación, y ser permeable a las influencias externas que propician transformaciones sociales a las cuales el sistema educativo debe atender. Por ello, la educación ambiental en la universidad no debe ser concebida como la conservación o la gestión de los recursos naturales; no debe ser un curso más del curriculum, debe ser considerada bajo un nuevo enfoque de las relaciones entre el hombre y su entorno. Su enseñanza debe estar basada en la experiencia; en la cual se utilizan los recursos humanos, naturales y físicos del entorno como un lugar donde se identifican relaciones, comportamientos y medios de supervivencia, y donde todo se encuentra interrelacionado.

La educación ambiental no se debe añadir a los programas educativos como una materia aparte o un tema concreto de estudio. Ella debe ser el resultado de la concertación de diferentes asignaturas y experiencias educativas, que permitan percibir el ambiente de manera global, universal; para así responder a las necesidades sociales. De ahí, que Leff (2004) señala que:

La educación ambiental implica un proceso de concientización sobre los procesos socio ambiental emergente, que movilizan la participación ciudadana en la toma de decisiones, junto con la transformación de los métodos de investigación, información desde una mirada holística y enfoques interdisciplinarios. (p.215)

En este contexto, la educación ambiental debe ser continua, para evitar que los conocimientos que se van adquiriendo queden obsoletos, y de esta manera asegurar la eficacia de las acciones a aprender. Por ello, se deben reformular constantemente los objetivos, contenidos, métodos; asegurando, día a día, la adaptación de dichos conocimientos en los individuos y grupos.

La importancia que ofrece una visión interdisciplinaria es romper la rigidez de las asignaturas tradicionales e integrar el contenido de los programas de estudio en un proyecto multidisciplinario relativo al ambiente; de tal manera que el educando pueda tomar 


\section{UNA PROPUESTA PARA LA DIDÁCTICA DE LA EDUCACIÓN AMBIENTAL EN LAS}

UNIVERSIDADES PÚBLICAS

Lisbeth Bermudez y Dilia Escalante de Urrecheaga

(pp 102-124)

conciencia de su contexto socio - natural, y que a su vez le permita modificar su entorno en la búsqueda de una mejor calidad de vida. Replantear el diseño curricular de la Educación Ambiental bajo un enfoque transdisciplinarios es una respuesta a la situación ambiental del mundo contemporáneo donde los procesos naturales y de la sociedad aparecen ya como fenómenos de una misma totalidad, y que es una exigencia del encuentro entre las Ciencias Naturales y las Ciencias Sociales.

Lo que se busca con ello, no es la operatividad de un discurso, sino realizar una reformulación profunda de los fines y medios del quehacer universitario a la luz de la preocupante situación ambiental actual, pues de lo contrario, los proyectos ambientales universitarios seguirán siendo simples paliativos. Es por ello, que el currículo debe estar en constante actualización que pueda servir de guía en el proceso de enseñanza y aprendizaje, más aún de un área del conocimiento como lo es lo ambiental, que requiere de una concepción amplia de los problemas que ha ocasionado el uso y abuso de la naturaleza para poder buscar las posibles soluciones que minimicen su impacto en el desarrollo de la vida en el planeta.

\section{Fundamentación epistemológica}

En el proceso de la producción de conocimientos intervienen distintas posiciones teóricas que, independientemente de la posición asumida, generan saberes que deben ser sometidos al estudio crítico reflexivo y analítico a fin de promover pensamientos y acciones estrechamente sincronizados con la dinámica ambiental y todas las situaciones problemáticas que de ella se derivan. Por ello, es insoslayable redefinir la relación que el hombre establece con la naturaleza en tanto ello tenga como propósito la alteración, explotación y agotamiento de los recursos naturales, como base o fundamento de un estilo de desarrollo depredador; situación que contradice el principio integrador hombre sociedad-naturaleza.

Volumen $22 N^{\circ} 1$ Enero-Abril 2018. ISSN: 2244-7296 


\section{UNA PROPUESTA PARA LA DIDÁCTICA DE LA EDUCACIÓN AMBIENTAL EN LAS}

UNIVERSIDADES PÚBLICAS

Lisbeth Bermudez y Dilia Escalante de Urrecheaga

(pp 102-124)

Dentro de este contexto, la educación ambiental, juega un papel relevante como paradigma válido que conduce a alcanzar una visión compleja de la realidad ambiental a través de una interpretación y la trascendencia que las acciones humanas pueden ocasionar a su entorno. De ahí que, si la educación ambiental pretende que las personas logren una visión amplia, compleja, holística y comprometida con la realidad deberá tener en cuenta la manera cómo adquieren los estudiantes un conocimiento contextualizado (e.g. valores, creencias, conocimientos previos, entre otros) para lograr adoptar a su vez una metodología que facilite la comprensión de los contenidos, y que de alguna manera pueda modificar en los discentes su conducta frente al ambiente. Por ello es pertinente cambiar la praxis pedagógica, considerando lo señalado por Pérez (1999):

Centrar la atención en las prácticas y matrices epistemológicas que se expresan en el proceso de producción de conocimientos vinculado a los problemas que se derivan de la acción antropogénica sobre los diversos ecosistemas y que, por supuesto, involucran a la educación ambiental. (p.5)

La construcción del conocimiento, para que pueda ser efectiva, debe ineludiblemente apoyarse en lo que piensan los sujetos con respecto al tema que se va a desarrollar, sea ese pensamiento acertado o erróneo conceptualmente. En tal sentido, la construcción del conocimiento sobre el ambiente es un proceso donde deben converger por un lado las cualidades y problemas del entorno, y por otro, los esquemas previsores o marcos de referencia que forman parte de la estructura semántica del individuo o del grupo que aprende. De esta manera se construirán aprendizajes significativos a partir de la interacción sujeto-entorno, que permiten a los estudiantes modificar y preparar nuevos aprendizajes. Así, el papel del educador ambiental es servir de mediador para el logro de una relación armónica hombre - naturaleza, que a su vez dará lugar a un nuevo conocimiento. Para ello, el docente o facilitador debe diseñar estrategias didácticas o acciones pedagógicas necesarias para que los profesionales en formación que cursan Educación Ambiental puedan relacionar lo nuevo que aprenden con lo que ya sabían, bien para confirmarlo, 


\section{UNA PROPUESTA PARA LA DIDÁCTICA DE LA EDUCACIÓN AMBIENTAL EN LAS}

UNIVERSIDADES PÚBLICAS

Lisbeth Bermudez y Dilia Escalante de Urrecheaga

(pp 102-124)

ampliarlo, modificarlo o cuestionarlo. Ideas que se complementan con Leff (2004) cuando expresa que:

Educar para formar un pensamiento crítico, creativo y prospectivo, capaz de analizar las complejas relaciones entre procesos naturales y sociales, para actuar en el ambiente con una perspectiva global, pero diferenciada por las diversas condiciones naturales y culturales que lo definen. (p.217)

Esto implica un desafío para los institutos de educación universitaria en cuanto a la producción de conocimiento a través de sus tres ámbitos de acción: docencia, extensión e investigación, y a su vez, por ser los sitios donde se generan las posibles soluciones a los problemas de la sociedad. En consecuencia, las universidades deben convertirse en espacios de construcción de esperanza para un futuro sustentable, desde una visión y comprensión integral del mundo y del ser humano que ayude a sus egresados a ser partícipes directos en la transformación de su entorno.

En la propuesta que presentamos se plantean objetivos concretos que pueden ser alcanzados con acciones específicas, donde se deben desarrollar temas puntuales, que pueden ser estructurados o reformulados según el contexto y realidad de cada institución universitaria. Todo ello, sin dejar a un lado el desarrollo de temas ambientales que sobrepasen el repetir una historia ambiental de congresos y conferencias ya conquistados; y que, por el contario, busquen dar una opción de repensar la Educación Ambiental, y abrir espacios para la reflexión de la actuación del hombre y su interacción con el ambiente, que puedan servir para confrontar ideas y llegar a conclusiones que puedan reorganizar esquemas conceptuales que despierten en los participantes la sensibilidad hacia su entorno.

Es por ello que la propuesta se basa en el constructivismo que permite un conocimiento contextualizado de la realidad que afecta directamente a cada uno de los individuos que conforman la sociedad, y que pueda internalizar que su accionar va a producir un efecto positivo o negativo en el ambiente, ya que él no puede considerarse como un espectador de la naturaleza porque forma parte de la dinámica ambiental. Aunado al desarrollo de 


\section{UNA PROPUESTA PARA LA DIDÁCTICA DE LA EDUCACIÓN AMBIENTAL EN LAS}

UNIVERSIDADES PÚBLICAS

Lisbeth Bermudez y Dilia Escalante de Urrecheaga

(рp 102-124)

concepciones filosóficas como el pragmatismo, progresismo y reconstruccionismo, que en su conjunto contribuyen a que los estudiantes se conviertan en seres activos y pensantes para el realce de los valores en los momentos de crisis que es cuando siempre se ven amenazados.

\section{Objetivo General.}

Proponer Acciones Estratégicas - Pedagógicas para la didáctica de la asignatura Educación Ambiental en las universidades públicas del estado Nueva Esparta.

\section{Objetivos Específicos.}

- Sugerir la praxis de estrategias integradoras para la Educación Ambiental en las universidades públicas del estado Nueva Esparta.

- Replantear en el diseño curricular el enfoque pedagógico de la Educación Ambiental impartida en las universidades públicas del estado Nueva Esparta.

- Fomentar actividades pedagógicas, donde se tomen en cuenta los enfoques epistemológicos de la Educación Ambiental.

- Propiciar encuentros institucionales de educación universitaria relacionados con el ambiente.

- Incentivar la apertura hacia la participación activa de la universidad frente a los problemas ambientales de la comunidad.

\section{Cuadro $\mathrm{N}^{\circ}$ 1: Acciones Estratégicas-Pedagógicas para la Educación Ambiental}

\begin{tabular}{|l|l|l|}
\hline $\begin{array}{c}\text { OBJETIVOS } \\
\text { ESPECÍFICOS }\end{array}$ & $\begin{array}{c}\text { ACCIONES ESTRATÉGICAS } \\
\text { - PEDAGÓGICAS }\end{array}$ & \multicolumn{1}{|c|}{ TEMAS } \\
\hline \multirow{2}{*}{$\begin{array}{l}\text { * Desarrollo de actividades } \\
\text { fuera del aula de clase (e.g. } \\
\text { charlas, talleres, visitas guiadas, } \\
\text { trabajos de campo e } \\
\text { investigación). }\end{array}$} & $\begin{array}{l}\text { *Fundamentación } \\
\text { epistemológica, sociológica y } \\
\text { pedagógica de la educación } \\
\text { ambiental. }\end{array}$ \\
& $\begin{array}{l}\text { * Participación de los } \\
\text { estudiantes en actividades extra- }\end{array}$ & $\begin{array}{l}\text { Eación ambiental y } \\
\text { participación ciudadana. }\end{array}$ \\
& \multicolumn{2}{|c}{ Modelos de desarrollo y } \\
\hline
\end{tabular}

Volumen $22 \quad N^{\circ} 1$ Enero-Abril 2018. ISSN: 2244-7296 


\section{UNA PROPUESTA PARA LA DIDÁCTICA DE LA EDUCACIÓN AMBIENTAL EN LAS}

UNIVERSIDADES PÚBLICAS

Lisbeth Bermudez y Dilia Escalante de Urrecheaga

(pp 102-124)

\begin{tabular}{|c|c|c|}
\hline $\begin{array}{l}\text { Sugerir la praxis de } \\
\text { estrategias integradoras de } \\
\text { educación ambiental }\end{array}$ & $\begin{array}{l}\text { académicas relacionadas con el } \\
\text { ambiente. Es decir, que no } \\
\text { formen parte de los contenidos } \\
\text { programáticos de la asignatura y } \\
\text { que se continúen durante toda su } \\
\text { carrera. } \\
\text { * Apertura de cátedras libres } \\
\text { relacionadas con el ambiente }\end{array}$ & $\begin{array}{l}\text { educación ambiental. } \\
\text { * Contaminación ambiental y } \\
\text { su impacto social. } \\
\text { *Brigadas conservacionistas. } \\
\text { * Multiplicadores en } \\
\text { educación ambiental. } \\
\text { * Juntas conservacionista. } \\
\text { * Movimientos ambientalistas } \\
\text { * Ambiente y participación. } \\
\text { * Legislación ambiental. } \\
\text { * Juegos ecológicos. } \\
\text { * Elaboración de proyectos } \\
\text { ambientales. }\end{array}$ \\
\hline $\begin{array}{l}\text { Replantear en el diseño } \\
\text { curricular el enfoque } \\
\text { pedagógico de la educación } \\
\text { ambiental impartida. }\end{array}$ & $\begin{array}{l}\text { * Discusión acerca de la } \\
\text { inclusión de la educación } \\
\text { ambiental en el currículo de } \\
\text { estas universidades. } \\
\text { * Planificación y ejecución de } \\
\text { talleres, charlas, mesas } \\
\text { redondas, con expertos en el } \\
\text { área ambiental y estudiantes } \\
\text { para redefinir el enfoque } \\
\text { pedagógico de ésta en el } \\
\text { currículo. } \\
\text { * Planteamientos de los } \\
\text { lineamientos pedagógicos en los } \\
\text { que se basa la educación } \\
\text { ambiental y su inclusión a nivel } \\
\text { universitario. } \\
\text { * Evaluación periódica de los } \\
\text { contenidos ambientales de los } \\
\text { currículos. }\end{array}$ & $\begin{array}{l}\text { *Finalidad de la educación } \\
\text { ambiental en las } \\
\text { universidades. } \\
\text { * Enfoques pedagógicos de la } \\
\text { educación ambiental. } \\
\text { * Educación Ambiental como } \\
\text { estrategia pedagógica para } \\
\text { desarrollar valores y } \\
\text { actitudes responsables frente } \\
\text { al ambiente. } \\
\text { *Finalidad de los currículos } \\
\text { en las universidades. } \\
\text { * Problemas ambientales, } \\
\text { locales y globales prioritarios } \\
\text { y sus posibles soluciones. } \\
\text { * Reflexionar sobre el } \\
\text { desarrollo sustentable, } \\
\text { ecoturismo, ecofolklore, } \\
\text { ecociencia. }\end{array}$ \\
\hline $\begin{array}{l}\text { Fomentar actividades } \\
\text { pedagógicas, donde se } \\
\text { tomen en cuenta los } \\
\text { enfoques epistemológicos } \\
\text { de la educación ambiental. }\end{array}$ & $\begin{array}{l}\text { * Planificación y ejecución de } \\
\text { actividades pedagógicas que den } \\
\text { lugar a la crítica reflexiva y al } \\
\text { enfoque interdisciplinario de la } \\
\text { educación ambiental. } \\
\text { * Planificación y ejecución de } \\
\text { actividades con enfoque } \\
\text { constructivista del conocimiento } \\
\text { ambiental. } \\
\text { * Proposición de estrategias por } \\
\text { parte de los estudiantes que }\end{array}$ & $\begin{array}{l}\text { * Análisis crítico-reflexivo de } \\
\text { material y/o situaciones } \\
\text { ambientales actualizadas. } \\
\text { * Realizar trabajos de campo } \\
\text { periódicamente en el } \\
\text { desarrollo de la asignatura de } \\
\text { educación ambiental. } \\
\text { * Realizar trabajos de } \\
\text { investigación donde se pueda } \\
\text { lograr la resolución de los } \\
\text { problemas ambientales del } \\
\text { entorno. }\end{array}$ \\
\hline
\end{tabular}

Volumen $22 N^{\circ} 1$ Enero-Abril 2018. ISSN: 2244-7296 


\section{UNA PROPUESTA PARA LA DIDÁCTICA DE LA EDUCACIÓN AMBIENTAL EN LAS}

UNIVERSIDADES PÚBLICAS

Lisbeth Bermudez y Dilia Escalante de Urrecheaga

(pp 102-124)

\begin{tabular}{|c|c|c|}
\hline & $\begin{array}{l}\text { puedan ser utilizadas en el } \\
\text { desarrollo de los contenidos } \\
\text { ambientales. }\end{array}$ & \\
\hline $\begin{array}{l}\text { Propiciar encuentros } \\
\text { institucionales de } \\
\text { educación universitaria } \\
\text { relacionados con el } \\
\text { ambiente. }\end{array}$ & $\begin{array}{l}\text { * Realización de encuentros } \\
\text { periódicos entre los institutos de } \\
\text { educación universitaria, } \\
\text { relacionados con el ambiente } \\
\text { (foros, coloquios, talleres, } \\
\text { jornadas, conversatorios). } \\
\text { * Registro en un compendio de } \\
\text { las propuestas, avances y } \\
\text { progresos alcanzados en los } \\
\text { encuentros ambientales a nivel } \\
\text { regional. } \\
\text { * Establecimiento de políticas } \\
\text { de control y seguimiento; tanto } \\
\text { en los contenidos como en la } \\
\text { orientación curricular } \\
\text { seleccionada. }\end{array}$ & $\begin{array}{l}\text { * El ambiente y la } \\
\text { universidad. } \\
* \text { La universidad y su } \\
\text { incidencia en la sociedad. } \\
\text { * Desarrollo sostenible y su } \\
\text { influencia en la educación } \\
\text { universitaria. } \\
\text { * Avances ambientales en } \\
\text { educación universitaria a } \\
\text { nivel regional. } \\
\text { *Globalización y } \\
\text { problemática ambiental } \\
\text { mundial. }\end{array}$ \\
\hline $\begin{array}{l}\text { Incentivar la apertura hacia } \\
\text { la participación activa de la } \\
\text { universidad frente a los } \\
\text { problemas ambientales de } \\
\text { la comunidad. }\end{array}$ & $\begin{array}{l}\text { * Ejecución de investigaciones } \\
\text { educativas basadas en estudios } \\
\text { directos con su entorno, } \\
\text { relativos a la problemática } \\
\text { ambiental. } \\
\text { * Abordaje de problemas } \\
\text { ambientales a través de la praxis } \\
\text { transdisciplinaria. } \\
\text { * Aprovechamiento del entorno } \\
\text { como el mejor recurso para la } \\
\text { aprehensión, interpretación y } \\
\text { solución de problemas } \\
\text { ambientales. }\end{array}$ & $\begin{array}{l}\text { * Trabajos de investigación } \\
\text { sobre problemas ambientales } \\
\text { de la región. } \\
\text { * Formas de participación } \\
\text { ciudadana comunitaria. } \\
\text { * Rol de las organizaciones } \\
\text { no gubernamentales (ONG) y } \\
\text { de los entes gubernamentales } \\
\text { en la problemática ambiental. } \\
\text { * Política ambiental oficial y } \\
\text { participación ciudadana. } \\
\text { * Integración de los consejos } \\
\text { comunales en los proyectos } \\
\text { ambientales desarrollados } \\
\text { por las universidades. }\end{array}$ \\
\hline
\end{tabular}

\section{CONSIDERACIONES FINALES}

El desarrollo de la propuesta que se presenta implica la consideración de algunos aspectos administrativos y curriculares; tales como:

Volumen $22 N^{\circ} 1$ Enero-Abril 2018. ISSN: 2244-7296 


\section{UNA PROPUESTA PARA LA DIDÁCTICA DE LA EDUCACIÓN AMBIENTAL EN LAS}

UNIVERSIDADES PÚBLICAS

Lisbeth Bermudez y Dilia Escalante de Urrecheaga

(pp 102-124)

- La didáctica de esta propuesta no puede limitarse a la simple transferencia de información o de contenidos. Los temas ambientales deben problematizarse desde una visión transdisciplinaria y multidimensional. De ahí, el valor que tiene la implementación colegiada de la propuesta con la participación de docentes de otras asignaturas o áreas (i.e. biología, áreas de la salud, historia, deporte, física, entre otras)

- La transformación se hará evidente si las acciones estratégicas - pedagógicas reflejan los pilares del proceso enseñanza - aprendizaje; es decir: aprender a ser, aprender a conocer, aprender a hacer, y aprender a vivir juntos; asociados además a las dimensiones universitarias de docencia, investigación y extensión.

- La programación debe ajustarse a las peculiaridades del currículo de cada carrera en cada institución (i.e Unidad Crédito y modalidad de escolaridad). Asimismo, se deben determinar las sesiones requeridas para el desarrollo de los temas o tópicos seleccionados y actividades de campo.

- La incorporación a contextos externos a las universidades (i.e. comunidades, parques, organismos públicos y privados) para el desarrollo de mini-proyectos.

- Los grupos de participantes deben ser de 20 estudiantes. Ello facilita la actividad grupal y actividades fuera de la institución.

- La revisión de los logros de los participantes no debe sólo indicar una calificación en función de conocimientos adquiridos, sino reflejar los valores y el accionar de los participantes ante la problemática ambiental.

\section{REFERENCIAS}

Arana, A. (1999). Ambiente y Sociedad... Un enfoque Integrador. Turmero, Aragua Venezuela: Instituto Pedagógico Rural El Mácaro.

Bermúdez, L., Escalante, D. y Figueira, A. (2016). Alcance conceptual y operacional de los programas de educación ambiental ofrecidos por las universidades públicas del estado Nueva Esparta. Academia. Vol. 15, No. 36, Julio - Diciembre. 


\section{UNA PROPUESTA PARA LA DIDÁCTICA DE LA EDUCACIÓN AMBIENTAL EN LAS}

UNIVERSIDADES PÚBLICAS

Lisbeth Bermudez y Dilia Escalante de Urrecheaga

(pp 102-124)

Caride, J. (2000, Octubre). Educación Ambiental y Desarrollo Humano: Nuevas Perspectivas Conceptuales y Estratégicas. Ponencia presentada en el III Congreso Iberoamericano de Educación Ambiental. Caracas - Venezuela.

Hidalgo, C. (2013). Gestión ambiental universitaria: Avances desde la Universidad Centroccidental Lisandro Alvarado. Ponencia presentada en el Seminario "La ULA en el paradigma de la universidad ambiental. Mérida, Junio 2013. [Documento en Línea]. Disponiblewww.eventos.ula.ve/ciudadsostenible/pdf_foro/DeCareliaUCLA.pdf[Consult a: 2017, Febrero 2].

Leff, E. (1998). Saber Ambiental. México. Siglo XXI editores, S.A.

Leff, E. (2004). Racionalidad Ambiental. La Reapropiación Social de la Naturaleza. México: Siglo XXI Editores.

Leff, E. (2014). Educación ambiental y filosofía de vida y construcción de mundos sustentables. [Documento en línea]. Ponencia presentada en la Universidad de Guanajuato, México. Disponible:

https://www.youtube.com/watch?v=z_9clHuKYgg[Consulta: 2017, Febrero 17]

Melendro, M., Novo M., Murga M. y Bautista M. (2009). Educación Ambiental y Universidad en la Sociedad de la Globalización. [Documento en Línea]. Disponible https://www,scielo.org.ve/scielo.php?script=sci_arttext\&pid=S1315. [Consulta 2017, Enero 16].

Morín, E. (1993). Tierra Patria. Barcelona: Editorial kairos.

Pérez, E. (1999). Pedagogía, Dominación e Insurgencia. Caracas- Venezuela: Ediciones Los Heraldos Negros.

República Bolivariana de Venezuela. Ley del Plan de Patria, Segundo Plan Socialista de Desarrollo Económico y Social de la Nación, 2013-2019. Gaceta Oficial de la República Bolivariana de Venezuela 6.118 (Extraordinario), Diciembre 4, 2013.

Révora, S. (2001). Formación ambiental universitaria. Ponencia presentada en la Jornada de Reflexión "Los desafíos de la educación ambiental universitaria. Buenos Aires, Junio 2001. [Documento en Línea]. Disponible https://www2.medioambiente.gov.ar/documentos/educacion/red_universitaria/formacion _ambiental_superior/reflexiones. [Consulta 2017, Febrero 6]. 
UNA PROPUESTA PARA LA DIDÁCTICA DE LA EDUCACIÓN AMBIENTAL EN LAS

UNIVERSIDADES PÚBLICAS

Lisbeth Bermudez y Dilia Escalante de Urrecheaga

(pp 102-124)

UNESCO (Noviembre 2014). Declaración de Aichi-Nagoya sobre la Educación para el Desarrollo Sostenible. Documento en Línea http://unesdoc.unesco.org/images/0023/002310/231074s.pdf. [Consulta 2017, Febrero 2]

Velásquez, F. y Fernández, M. (1998). Temas Ambientales en las Ciencias de la Vida. Madrid: NARCEA, S.A 